\title{
IMPLEMENTATION OF A NEW METHOD \\ TO TRACK PROPOFOL IN AN ENDOSCOPY UNIT
}

\author{
A Scholarly Project \\ submitted to the Faculty of the \\ Graduate School of Arts and Sciences \\ of Georgetown University \\ in partial fulfillment of the requirements for the \\ degree of Doctor of Nursing Practice
}

By

Catherine Horvath, M.S.N.

Washington, D.C.

October 27, 2016 
Copyright 2016 by Catherine Horvath

All Rights Reserved 


\title{
IMPLEMENTATION OF A NEW METHOD TO TRACK PROPOFOL IN AN ENDOSCOPY UNIT \\ Catherine Horvath, M.S.N. \\ Thesis Advisor: Peggy Compton, Ph.D.
}

\begin{abstract}
Propofol is a widely used anesthesia induction agent and is easily accessible in most healthcare facilities. Unlike regulated or schedule drugs, propofol is inconsistently tracked, leading to inaccurate inventories, incorrect billing, and unrecognized diversion. The goal of this project was to implement a new method of tracking propofol in a single setting, with the aim of accounting for $100 \%$ of the drug. For two, 2-week observation periods, data was extracted from an automated medication management system or pharmacy inventory system, anesthesia records, and pharmacy billing sheets for cases in a GI Endoscopy unit, and compared pre- and postimplementation of a new tracking and accounting protocol. Variables included amount of propofol (a) inventoried; (b) dispensed; (c) administered; (d) returned; (e) billed; (f) wasted, and (g) missing. Pre-implementation $(\mathrm{n}=300), 10 \%$ cases had no record of propofol removal from the automated medication system. Of the $90 \%$ that did, $25 \%$ had an amount signed out that did not match the amount administered. Thirty-one percent of all propofol was unaccounted for during this 2 -week period. Further $19.7 \%$ of cases did not have a billing form located. Postimplementation of the process change, unaccounted for propofol decreased to $26 \%$ of all cases $(p=n . s)$. The percentage of missing billing forms decreased from $19.7 \%$ to $5.8 \%(p=0.00)$. Data suggest notable amounts of propofol were unaccounted for and not billed for in the GI Endoscopy unit prior to the protocol change. Post-protocol change data reveals modest improved accounting of the medication and significant cost-capture. Improvements may enhance
\end{abstract}


inventory reconciliation, availability of drug stock, and potentially result in a decreased risk of unrecognized diversion. 


\section{ACKNOWLEDGEMENTS}

Peggy Compton, mentor

Nancy Crowell, statistician

Donna Clarke and Dinise Barrow, pharmacy

Medhat Hannallah, clinical champion

\section{DEDICATIONS}

This thesis is dedicated to my family, who believe in me. 


\section{TABLE OF CONTENTS}

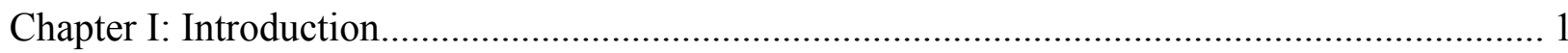

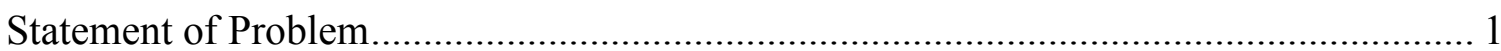

Background and Significance …………...................................................................... 1

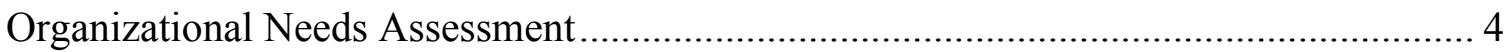

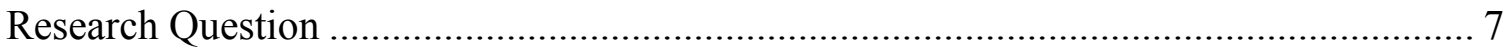

Evidence Based Practice Model of Implementation......................................................... 8

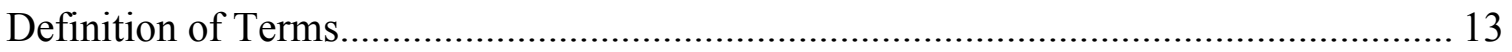

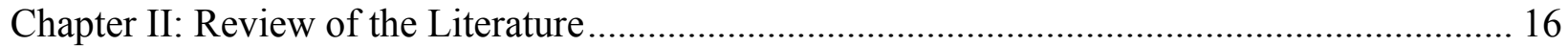

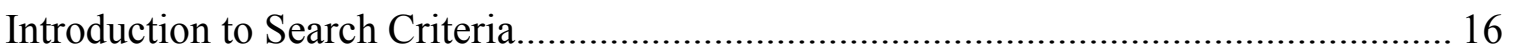

Critique and Synthesis of Previous Evidence …………….......................................... 18

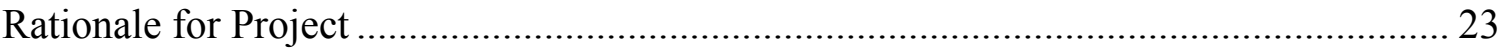

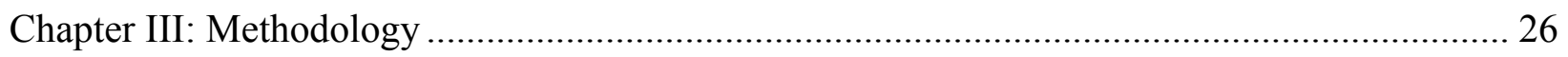

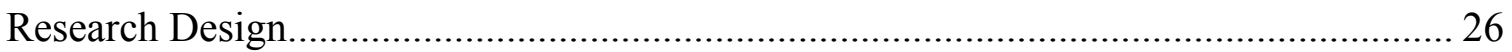

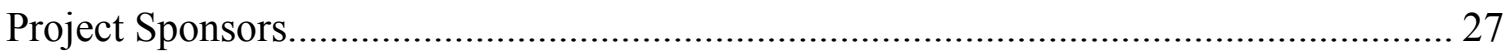

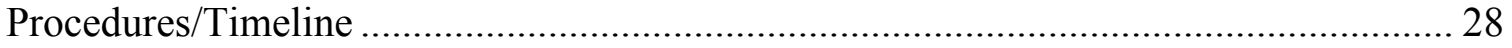

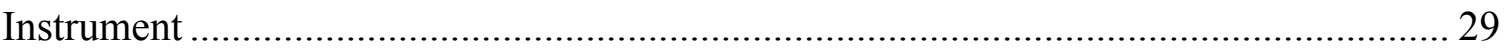

Protection of Human Subjects ....................................................................................... 30

Population Sample ………………………………………………………………... 30

Outcome Measures/Data Analysis Plan........................................................................... 31

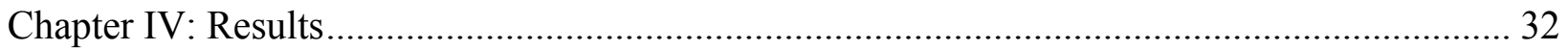

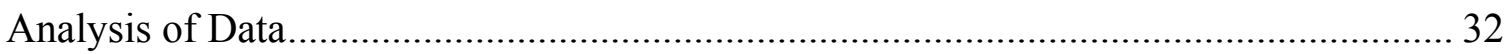

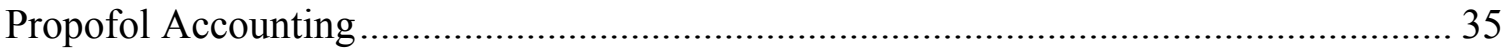

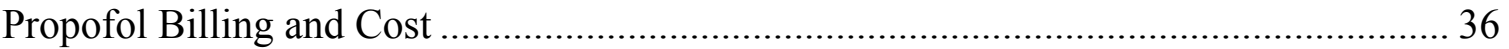




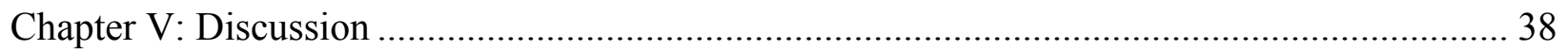

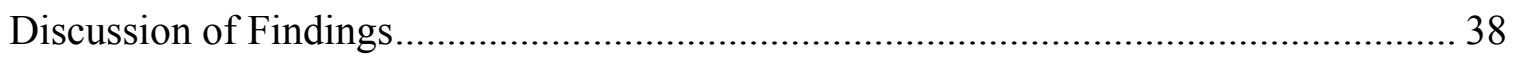

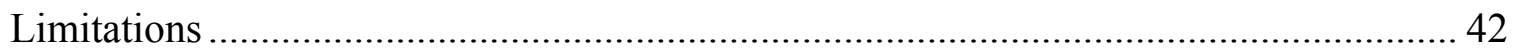

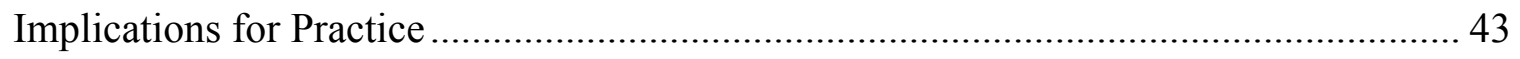

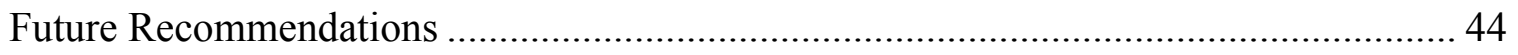

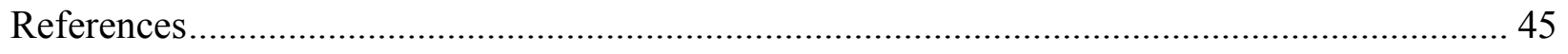

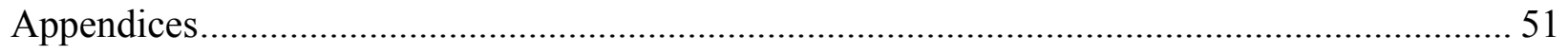

Appendix A: Iowa Model of Evidenced-Base Practice to Promote Quality Care............ 51

Appendix B: GI Endoscopy Unit Propofol Policy .......................................................... 52

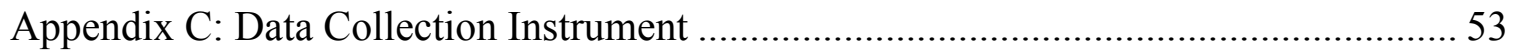

Appendix D: Revised Pharmacy Billing Form ............................................................. 54 


\section{LIST OF TABLES}

Table 1: Decision tree for study inclusion in systematic review of propofol tracking methods .. 17

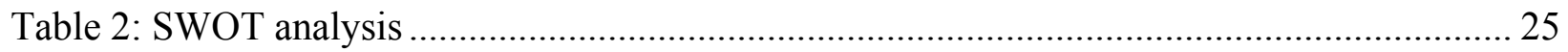

Table 3: Sample and case demographics, pre-and post-intervention...................................... 34

Table 4: Propofol accounting and loss, pre- and post-intervention ........................................ 35

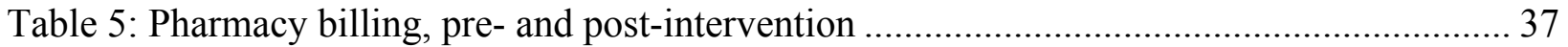




\section{Chapter I}

\section{Introduction}

\section{Statement of Problem}

Propofol (Diprivan ${ }^{\circledR}$, Fresenius Kabi, Inc.) is the most widely used anesthesia induction agent and intensive care unit sedative in the world (Monroe, Hamza, Stocks, Scimeca, \& Cowan, 2011). As a drug not scheduled under the Controlled Substance Act, propofol is easily accessible and poorly controlled in most healthcare facilities (U.S. Department of Justice: Drug Enforcement Administration, 2010). Problems can arise from lax accounting. The drug can be lost and not available for patient usage, exacerbating shortages. Inventory may be inaccurate resulting in unavailable stock. Healthcare workers may divert drug for self-administration, which can impact provider and patient safety, as well as institutional reputation. Healthcare costs increase due to lost or stolen drug. These significant issues call for the implementation of methods to rigorously track propofol within a healthcare facility.

\section{Background and Significance}

Propofol is a non-barbiturate hypnotic drug with a rapid onset of action and recovery profile (Roussin, Montastruc, \& Lapeyre-Mestre, 2007). It is reported to produce pleasant feelings of calm, euphoria, and positive dreams. It leaves little to no residual cognitive effects. Propofol is generally recognized to be safe when used under the supervision of a qualified professional, although serious side effects such as cardiovascular and respiratory depression can occur even with normal dose administration. These conditions may require artificial support, however return to baseline status is remarkably rapid and the effects quickly resolve when drug 
administration stops. Related to its primary indications for initiation and maintenance of general anesthesia, anesthesia providers are high utilizers of the medication.

Propofol is currently not a scheduled drug with the Drug Enforcement Administration (DEA) under the U.S. Controlled Substance Act (U.S. Department of Justice: Drug Enforcement Administration, 2010). On the grounds that addictive properties were not recognized in clinical trails, propofol was approved by the Food and Drug Administration (FDA) and introduced to the market in 1989 unscheduled (U.S. Food and Drug Administration, 2016). However, as time has passed, documented cases of misuse and abuse have been steadily increasing, many ending in death. In $96 \%$ of these cases, the abusers were health care providers or were in training to become health care providers (U.S. Department of Justice: Drug Enforcement Administration, 2010). In 2010, the DEA proposed changing propofol's schedule status but the rule did not pass. Two states, Alabama and Georgia, have recognized the risks related to misuse and currently have laws that schedule propofol as a controlled substance (Alabama Department of Public Health, 2012; Georgia Drugs and Narcotics Agency, 2016).

A scheduled drug carries special requirements for acquisition, possession, storage, prescribing, dispensing, supplying, administering, and recording. As propofol is not regulated under these rules, monitoring of storage and accounting need not be strict. Several issues arise with the relative casual control of propofol. For one, inventory in a specific setting becomes sporadic. Practitioners may acquire more drug than needed, and not return them, leading to displaced or lost drug, or drug not being in the expected location because it is not returned to its proper place. When the drug is not readily available, it can lead to delays or interruptions in patient care. Missing supply can also contribute to higher costs to the institution by necessitating the need to replace the drug to maintain inventory. 
In addition to local institutional scarcity, lenient control is related to national drug shortages. National shortages can occur with any drug. The FDA maintains a real-time database that lists drugs currently in short supply (U.S. Food and Drug Administration, 2016). Sterile injectable drugs appear on the list the most, due to the complexity of the manufacturing process for sterile injectable solutions, quality issues, and a limited number of production lines that can make the drug. Propofol, a sterile injectable drug, is particularly susceptible to shortages due to its distinctive solution components (Jensen \& Rappaport, 2010). Data collected by the Drug Shortage Program of the FDA shows that propofol has been on the national drug shortage list multiple times since 2009, when manufacturing issues closed several plants, with the last major shortage noted in 2014 (Hvisdas, Lordan, Pizzi, \& Thoma, 2013; American Society of HealthSystem Pharmacists, 2016). Drug shortages can negatively impact the quality of patient care by causing alterations of treatment plans, substitution of less efficacious or more dangerous medications, inappropriate handling of drug, and cancellation of procedures (Osby, 2014).

Perhaps of most concern, warnings of misuse and abuse of propofol have been repeatedly reported in the literature since its release into the market in 1989. Best estimates report the percentage of healthcare providers with substance use disorder of all types of addictive pharmacology is from 2-10\% (Bell et al., 1999), with the percentage in anesthesia providers specifically as high as $19 \%$. A variety of drugs can lead to substance misuse. Regardless of the root drug of a provider's addiction, of those providers in substance abuse treatment programs, nearly $33 \%$ reported using propofol at least once in the course of their disease (Stocks, 2011). Within anesthesiology training programs in the United States, 18\% percent report experiencing at least one, if not more, cases of propofol diversion or abuse in the past 10 years; $71 \%$ of these programs did not have a system to secure and account for propofol within the institution 
(Wischmeyer et al., 2007). The American Association of Nurse Anesthetists cites the rate of substance use disorder of certified registered nurse anesthetists at more than $15 \%$ and provides evidence that the misuse of propofol is on the rise (Luck \& Hendrick, 2004). Almost all cases of propofol diversion and abuse involve healthcare providers or those in professional training programs, due to availability limited to intensive care units and surgical settings. Highly concerning is that $28 \%$ of these abusers died due to a propofol overdose (U.S. Department of Justice: Drug Enforcement Administration, 2010). Health care workers with substance use disorders are a significant concern for both the health care worker's well-being and the public's safety. The most common reason noted by providers with addiction issues for choosing a particular drug for misuse is its ease of access to the drug (Stocks, 2011). Therefore methods to limit access might deter misuse.

There are many arguments against controlling access to propofol in the clinical setting. Doing so may lead to delays in obtaining the drug in urgent or emergent situations, and accounting for each unit of propofol is too burdensome due to the time associated with its frequent use (Stocks, 2011). Stricter pharmacy control would entail increased cost and more administrative oversight. Since controls are not mandated or regulated, pharmacy departments may not appreciate the value of access restriction and therefore challenges institutional buy-in.

To address the issues associated with propofol shortages, waste, and the risk of provider misuse, this project will focus on developing a new method to track propofol to prevent loss, capture cost, and possibly detect drug diversion activities in a healthcare setting.

\section{Organizational Needs Assessment}

The organization is a large urban teaching hospital located in the Mid-Atlantic region. The hospital employs approximately 4,000 people, of which about 700 are registered nurses and 
300 are physicians, and boasts Centers of Excellence in Neurosciences, the Transplant Institute, Gastroenterology and the Lombardi Comprehensive Cancer Center. The hospital is part of a healthcare network that includes 10 hospitals and numerous outpatient and primary care settings, and is the largest nonprofit healthcare system in the mid-Atlantic region.

The Department of Anesthesiology at the hospital is a service department comprised of approximately 25-30 board-certified physician anesthesiologists (MDAs), 20-25 certified registered nurse anesthetists (CRNAs), 24 anesthesia residents, 4 registered nurses (RNs), 12-14 student registered nurse anesthetists (SRNAs), 6 anesthesia technicians, and 6 administrative staff members, totaling over 100 members. The department is led by a chairman, with the daily managing duties shared by 3 rotating MDAs. A senior CRNA serves as chief CRNA, a staff CRNA serves as clinical coordinator for the SRNA students, and one MDA serves as residency director. The department members are typically not found in offices, but spread out over 32 anesthetizing sites throughout the hospital, including operating rooms and satellite locations. Members provide anesthetic services for all surgical subspecialties to approximately 20,000 patients per year (including pediatric and high-risk obstetrical patients). The department members also provide acute and chronic pain services, and critical care medicine in the surgical intensive care unit.

Being the most common anesthetic drug in the world, propofol is widely used within various units of the hospital, with the central Pharmacy accounting for over 2.3 million milliliters per year. As is the case in many health care facilities across the country, this hospital has permissive control over propofol. The drug is purchased, stored, and distributed throughout the institution by the Pharmacy department. For anesthesia purposes in the operating room and anesthetizing areas outside the operating room (interventional radiology, bone marrow harvest, 
obstetrics), propofol is kept stocked and secured in anesthesia carts. However, in the GI Endoscopy Unit, pharmacy dispenses the drug into an automated medication management system (Pyxis Medstation ${ }^{\circledR}$, Cardinal Health Inc.) for storage. Although the management system has the capability to identify the individual patient for whom the drug is signed out, the provider withdrawing the drug is not required to identify how it is used and, limited crosschecking of the quantities disbursed and the amounts used for each patient is done. The pharmacy counts propofol for inventory and cost only; there is no attempt to monitor use by providers or track handling patterns of the drug. Therefore, diversion is difficult to track. A common practice is for anesthesia providers to access large quantities of propofol at the beginning a shift to use throughout the day. The amount of propofol actually used for patient care is documented on a permanent patient record. A separate paper-billing sheet is filled out to correspond to the number of propofol vials opened for each patient. But the current billing methods and medication management system do not capture if the propofol is actually used, and what happened to any excess signed out.

This study is to take place in the Gastroenterology Endoscopy Unit within the hospital. The unit is comprised of six procedure rooms, a preoperative holding area, and a postoperative recovery area. Approximately sixty-eighty healthcare providers work there on a given day. There is one gastroenterologist that oversees the entire unit, with one MDA who specifically manages the anesthesia team. On any particular day, there are 2-3 MDAs in charge, and 4-6 anesthesia residents, SRNAs, or CRNAs. The GI Endoscopy Unit was chosen for this project due to its almost entire propofol-based anesthesia, numerous procedures per day, and high patient volume necessitating the handling of large quantities of propofol.

In the GI Endoscopy Unit, propofol is dispensed from one Pyxis Medstation located in 
the postoperative area. Drugs can only be dispensed to licensed providers, usually anesthesia providers. Anesthesia providers are instructed to sign out propofol to each individual patient assigned to him/her. However, this is not always done and taking many vials at one time may occur with the system open. This allows extra propofol to be signed out to one patient, but to be used for another patient later in the day. Therefore the recording of the propofol under the correct patient name does not always occur in the automated medication management system. Unused propofol is not returned to the Pyxis Medstation, it is left in the anesthesia carts or placed in a pharmacy return bin.

\section{Research Question}

A well-structured Evidence-Based Practice (EBP) project begins with the construction of the research question. A helpful format to use is the Patient, Intervention, Comparison, and Outcome (PICO) model (Poe \& White, 2010). The model develops a clear problem question, which launches the approach used to identify core keywords and search the evidence. The PICO for this project is as follows:

P: The drug propofol is not being tracked in the endoscopy unit.

I: Implementation of a new method to track propofol inventory and usage

C: The hospital's current methods of tracking inventory and usage with other healthcare institutions and published guidelines will help define best practices.

O: $100 \%$ of propofol is accounted for as dispense, wasted, administered, or returned and then correctly billed. Indirect outcomes: decrease lost revenue, improved availability, and decrease the risk of diversion.

Combining the elements of the PICO statement form the clinical practice question for the research project: In the Endoscopy unit at the hospital, will a new method of tracking and 
accounting for propofol inventory in the GI Endoscopy unit account for $100 \%$ of the propofol dispensed from pharmacy, including drug administered, waste, and return inventory, as compared to the current method of tracking and accounting for propofol? The hypothesis is that tracking $100 \%$ of propofol in the GI Unit will decrease propofol loss, serving as a proxy for decreased cost to the institution, decreased risk of shortages, improve billing capture, and decreased risk of diversion of the drug.

\section{Evidence Based Practice Model of Implementation}

For quality patient care, it is imperative that clinical health care be the best it can be, and be based on scientific evidence. However, changing a clinical practice can be complex and quite challenging. For clinicians implementing evidence-based practice (EBP), using a developed model to systematically guide the implementation can help with changing and sustaining new practices in their organizations (Melnyk \& Fineout-Overholt, 2011).

The Iowa Model of Evidence-Based Practice to Promote Quality Care is the model of EBP utilized at MedStar Georgetown University Hospital. The model outlines the change process as a series of problem-solving steps and feedback loops (see Appendix A) (Titler, Steelman, Budreau, Buckwalter, \& Goode, 2001). Health-related EBP researchers working with a multidisciplinary healthcare team benefit from its generalized applicability and ease of use with project development and implementation (Melnyk \& Fineout-Overholt, 2011).

The Iowa model begins with the identification of a practice question, which comes from questioning current practice. Is the poor accountability of propofol a problem for the nurse anesthesia practice in the GI Endoscopy Unit? The issues of cost, drug availability, and patient and provider safety surrounding proper medication use make this topic a priority for the organization. This issue also fits within the organizational priorities of care of the whole person 
and patient safety. Anesthesiology is recognized as the leading specialty in health care in addressing patient safety, so interest in this safety-related research is expected (Gaba, 2000). It is crucial to obtain support, including financial and personnel resources, from leadership and stakeholders, to carry out the practice change. A recent Department of Health visit to the institution cited that medications, including propofol, were not properly stored when not being used, further confirming the need for this project.

Once there is agreement from the stakeholders the topic needs to be addressed, the next step in the model is to form a team. This team will develop, implement, and evaluate the practice change (Melnyk \& Fineout-Overholt, 2011). It also provides support, varied viewpoints, and credibility to the project ideas. The stakeholders for this project include members from anesthesia, gastroenterology, pharmacy, nursing, and hospital administration. The team's goal will be to write a new policy and seek input and buy-in from senior leadership for this quality improvement project.

Next, relevant literature and other sources of information surrounding the topic are assembled, critiqued and synthesized. This review provides an evaluation of the published studies illustrating how the subject has been previously examined and provides a theoretical basis for the research project. As will be described, a thorough literature search and review for this project yielded low-quality evidence primarily in the form of case reports, as no high-quality research evidence was found for this topic.

The lower-level evidence will be used to pilot, and provide data to support a practice change. The pilot will determine if the project is feasible and whether the EBP practice change has the potential to be effective with respect to propofol accounting. According the Iowa model, trialing the EBP change with a pilot run is essential for finding issues before commencing with 
an institution-wide practice change (Melnyk \& Fineout-Overholt, 2011). The pilot phase will include designing a draft practice protocol, including an evidence-based model, procedure, methods, or other documentation outlining the practice and decision points. This project will be trialing such a new process.

Lastly, the Iowa model discusses an evaluation of the outcomes of the pilot to determine if there should be adoption or modification of the practice. If the change is deemed appropriate, the team leader institutes the change institution-wide. Monitoring and analyzing the process and outcome data will continue. Outcome data from this pilot study will determine if $100 \%$ of the propofol stocked in the GI Unit can be accounted for daily. Finally, it is crucial to disseminate the results of the project, so best practices can be shared and incorporated in other practice settings. It is planned that this project will be published in a peer-reviewed pharmacy, anesthesia, and/or nursing journal and presented at a national nurse anesthesia conference.

The Iowa model provides a guide to clinicians on developing and implementing EBP process change. The model includes several "feedback loops, reflecting analysis, evaluation, and modification" based on the success and/or failure that occur in the process and what the outcomes happen to be. These mechanisms help individualize the discovered evidence to a specific practice setting and aids in adoption of the change (Melnyk \& Fineout-Overholt, 2011).

Relevance to Nursing. The impact and implications of propofol accountability for nursing in the clinical setting are numerous. CRNAs and SRNAs, as advanced practice nurses, are at elevated occupational risk of developing substance misuse problems due to their daily exposure to addictive medications. Bedside nurses are also at risk, although at a low rate. Unit nurses may be the vigilant observers recognizing signs of an impaired provider or witnessing patient symptoms that could be the outcome of an impaired anesthesia provider. Nurses are on 
the front line, involved in the reconciliation of medications and can alert others of possible diversion. Because healthcare workers are given the responsibility for handling and administering drugs to patients, the impact of substance abuse among these individuals carries great consequences. It affects the health of patients, the health of the abusers themselves, the health of their fellow healthcare workers, and introduces risk to their employers (Berge, Dillon, Sikkink, Taylor, \& Lanier, 2012). Nursing is in a vulnerable position related to substance abuse, and for the health of the profession, it needs to take a strong stance to deal with the issue and help nurses recover.

Change theory. This project will be to implement a change in practice by developing a new policy surrounding the accountability of propofol. But to begin any change process, it is important to understand how to make change happen successfully. A fundamental model for conceptualizing organizational change was developed by Kurt Lewin and is known as the Unfreeze- Change- Refreeze model (Lewin, 1947).

The Unfreeze stage of change involves determining what needs to change within the organization and working to get everyone to accept that the old ways of doing things could be improved and therefore change is necessary. This can necessitate breaking down of the present state of conditions before developing a new way of operating. The key to unfreezing the status quo is crafting a compelling message outlining why the current processes should not continue. All members of the anesthesia department and GI staff place a high value on patient safety and quality patient care. Highlighting the inefficiencies and dangers of the current practice helps breakdown the status quo. Including all team members in any educational sessions and messaging the importance of the changes to everyone simultaneously will maximize participation. Communicating the vision and strategy of the project based on patient and 
provider safety and wellbeing as supporting evidence of why change should occur is imperative to break down walls.

New patient safety initiatives and policies are frequently distributed and discussed in departmental meetings but are not always followed. Therefore it is vitally important to remember the culture when designing education sessions or implementing new policies. As stated previously, anesthesiology is recognized as the leading specialty in health care in addressing patient safety (Gaba, 2000). Compared to decades earlier, the anesthesia profession has made great strides in making anesthesia care much safer by incorporating new technologies, standards, and guidelines, and addressing human error and systems issues. Anesthesia providers learn in training and practice to minimize risk and develop patient care strategies to improve safety (Haller, 2013). This makes anesthesia providers by training ritualistic people. These rituals can be observed in all the anesthetizing sites as demonstrated daily by proper machine checkouts, precise medication preparation, equipment placed within easy reach, and charting to reflect minute-to-minute fluctuations. The rituals make anesthesia efficient, routine, and safe, but also engender resistance for providers to change. Understanding the beliefs, values, attitudes, and behaviors of the department and providers that currently frame this issue will be crucial to the project's success.

After the uncertainty created in the Unfreeze stage, the Change stage is when new ways to accomplish a goal are considered, and others begin to flirt with ways to support and assist the implementation of actions that move the organization in a new direction. It is important to remind others how the change will benefit them by communicating often and preparing them for what is coming. The goal is to create a sense of urgency. For this project, face to face educational sessions, email correspondence, and other means of communicating the policy 
change will be implemented, as well as check-ins with the department members to get feedback. Involving people in the process will empower them, while generating short-term wins that will reinforce the change. There is a provider champion, a MDA, at the clinical site who will check on the process frequently. There will also be a midterm progress report given to the providers. This process will be reinforced by the involvement of four departments: pharmacy, anesthesia, nursing, and gastroenterology that will create synergy to keep the project moving along.

When the changes have become ordinary behavior and everyone has incorporated the new ways of working into their daily routine, the organization is ready to solidify the change in the Refreeze stage. The change becomes anchored into the culture and the institutional way of doing things. In part, this will be achieved with the development of a new institutional policy that is well publicized and supported by the institution. Frequent reinforcement of the process continues with email reminders, and occasional check-ins at staff meetings with the providers to get feedback. The provider champion on the unit will provide visible support and keep everyone informed of how the change is working on a weekly basis. Continuing education sessions to reinforce behavior are important will occur with anesthesia, nursing and pharmacy members. Identifying what supported the transition and what barriers still exist are imperative to improving and sustaining the change.

\section{Definition of Terms}

This project is evaluating the relationship among the concepts of propofol accounting practices, loss, and the cost to the institution. The hypothesis is that improving propofol reconciliation will decrease the loss of medication and decrease the financial cost, and ultimately decrease the risk of provider diversion of the drug for misuse through better tracking. For the purpose of this study, the following terms and definitions will be used: 


\section{Conceptual Definitions.}

1. Propofol accounting- reconciliation of propofol inventory on unit as dispensed, administered to the patient, documented as wasted, returned unused, or remaining in the medicine management system.

2. Propofol loss- propofol that is unaccounted for in the reconciliation process.

3. Cost to institution- institutional financial loss on unaccounted for propofol and propofol not billed correctly.

\section{Operational Definitions.}

1. Propofol accounting- processes by which the amount of propofol (in mg or vials) inventoried on the unit, is either documented as dispensed, administered, wasted, or returned.

2. Propofol loss- amount of propofol (in mg or vials) missing from inventory that was not dispensed, administered, wasted, or returned.

3. Inaccurate billing- pharmacy billing sheets that were either not returned to the pharmacy (missing) or had inaccurate billing recorded.

Beyond the conceptual and operational terms, other terms requiring definition are:

- Medication management system or automated medication dispensing system- "drug storage devices or cabinets that electronically dispense medications in a controlled fashion and track medication use" (Murray, 2001).

- Drug shortage- "a situation in which the total supply of all clinically interchangeable versions of an FDA regulated drug is inadequate to meet the current or projected demand at the user level" (Hvisdas, Lordan, Pizzi, \& Thoma, 2013, p. 171). 
- Substance use disorder (Diagnostic and Statistical Manuel of Mental Disorders-V definition)- the pathologic "recurrent use of alcohol and/or drugs that cause clinically and functionally significant impairment, such as health problems, disability, and failure to meet major responsibilities at work, school, or home. It is passed on evidence of impaired control, social impairment, risky use, and pharmacological criteria" related to tolerance or withdrawal (Substance Abuse and Mental Services Administration, 2016).

- Drug diversion- "the diversion of licit drugs for illicit purposes. It involves the diversion of drugs from legal and medically necessary uses towards uses that are illegal and typically not medically authorized or 'necessary' " (Center for Medicare \& Medicare Services, 2016). 


\section{Chapter II}

\section{Review of the Literature}

A comprehensive search and review of the literature related to this topic was performed. The literature review confirms that many policies and procedures to prevent drug loss and diversion have been implemented in healthcare facilities across the United States, most notably for scheduled drugs such as opioids. However, fewer reports address methods to prevent propofol loss and diversion specifically. Acknowledging the scope of the problems associated with poor propofol accounting practices, the findings of these reports and studies were critiqued and synthesized to develop an intervention to improve methods to track and secure propofol.

\section{Introduction to Search Criteria}

Using the electronic databases MEDLINE, Google Scholar, and Embase, the terms propofol, controlled substance, accountability, diversion, and prevention were searched alone or together. Articles reporting sources of data evaluating propofol and/or controlled-substance tracking methods published from 1980-2015 and in the United States or Canada were sought. References of the retrieved articles were also screened for additional studies. The online database literature search was supplemented by hand-searching reference lists from identified articles. Twenty articles were selected for the final systematic review (see Table 1). 
Table 1. Decision tree for study inclusion in systematic review of propofol tracking methods.

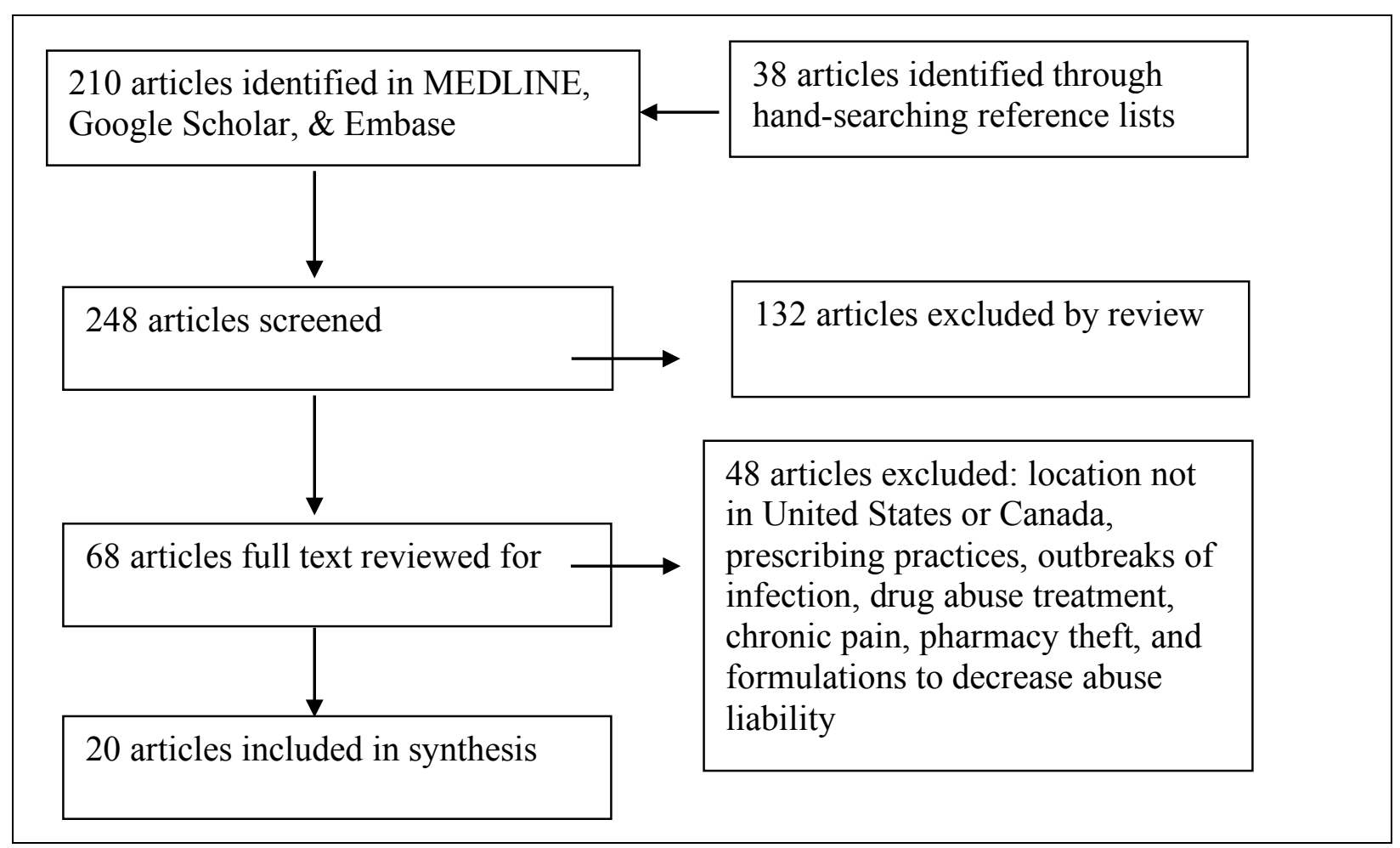

Titles and abstracts from the results of the initial searches were reviewed according to the established theme elements to determine appropriateness for inclusion. Articles addressing either propofol or controlled substances were included, as the regulated processes for tracking controlled substances versus uncontrolled-substances were important to understand. The remaining full-text articles were read. Forty-eight articles were excluded due to unrelated topic of article or study: articles consisting of a description of prescription practices only, concerns over outbreaks of infection, drug abuse treatment, chronic pain, pharmacy theft, medication formulation development to decrease abuse liability, and location outside the United States or Canada. Of the twenty remaining papers, six descriptive studies by Epstein, Gratch, and 
Grunwald (2007), Epstein, Gratch, McNulty, and Grunwald (2011), Kamerman, Quirk, and Bluml (1992), McClure, O’Neal, Grauer, Couldry, and King (2011), Schmidt and Schlesinger (1993), and Vigoda, Gencorelli, and Lubarsky (2007) provided the strongest level of evidence. All others were case reports, or observational studies. No meta-analysis, randomized controlled trials (RCTs), cohort studies, correlational studies, or clinical guidelines on the subject of propofol and accountability were found during the search.

\section{Critique and Synthesis of Previous Evidence}

Critical appraisal of this literature resulted in the development of a table of evidence. Rapid critical appraisal "checklists" were used to evaluate the validity and reliability of the study, and applicability of the findings to clinical practice for each of the studies (Melnyk \& Fineout-Overholt, 2011). The body of evidence identified was all lower-level evidence, making transferability and the reliability of intervention difficult. All studies had the goal to improve control of controlled substance and/or propofol access as a means to improve drug accountability and prevent diversion.

The six articles that produced the strongest evidence were evaluated. Two of the papers were from Epstein and colleagues $(2007 ; 2011)$. The first (Epstein, Gratch \& Grunwald, 2007) was a descriptive study using a data-mining approach. Identified indicators of diversion were searched for in an information system database, drug transaction logs were reconciled with the anesthesia patient records, and identifying occurrences of atypical drug transactions were pinpointed from the automated drug dispensing system. The institution had on record two known cases of drug diversion, and the study was to determine if this data-mining approach would reveal the individuals, which it did. Diversion of controlled substances (opiates and midazolam), not propofol, was studied, and data was collected retrospectively for an 11-month 
period. Analysis was done using a nonparametric graphical approach and controlled for outliers in the data. The resulting predictor model was able to correctly reveal the individuals who had diverted drug. The study identified limitations around methodology and transferability issues. There were only two index cases (known cases of diversion) and the data has not identified any new cases. Therefore, applying this method of data mining needs to be validated with future studies.

In follow-up to the first, Epstein, Gratch, McNulty, and Grunwald (2011) repeated their descriptive study, this time prospectively looking at the ability of their predictor model to identify diversion mining the same two databases described in the first study. Two previously unsuspected individuals were prospectively identified as diverting drugs, and suspicion rose for an additional individual, found to have careless documentation practices. This study validated a data-mining technique for screening for atypical drug transactions. However, investigators described weaknesses in the model, in that it is likely to have missed individuals diverting small amounts of drugs, work infrequently in the institution, or exploit methods outside the scope of the outlined process (i.e. mismatched anesthesia record and drug dispensing logs, falsifying records, etc.). Interestingly, the researchers noted they could not replicate the study with propofol due to issues with stocking the drug in their drug dispensing systems, the large amounts of utilization and wastage, and the wide variation in dosing and mode of administration (bolus or infusion) by clinicians.

In an early study, Kamerman, Quirk, and Bluml (1992) described a quantitative analysis method to investigate controlled substance theft or tampering on a nursing unit. The researchers outlined a simple "checkbook" system of accountability, looking at the total controlled substance usage over a given period of time for any unusual events or outliers. Two of the three steps 
described were implemented. They found that providers who sign out controlled substances "with less than honorable intent" frequently fail to accurately chart the dose in the patient record, as opposed to medication loss or diversion. No data was presented, making it difficult to ascertain the validity or reliability of the reported process. The authors noted the excessive time it took to utilize the process as a barrier to adoption.

At about the same time, Schmidt and Schlesinger (1993) reported on a prospective descriptive study implementing a new system of recording-keeping to account for the amount and type of drugs dispensed, administered, wasted, and returned. Controlled substances were studied (fentanyl, thiopental, and midazolam). In the first six months of the program, 37 cases of deviation occurred, but all were explained by either forgetting to return or fill out the proper forms, accidently wasting drug instead of returning, and drug left unattended. Two cases had anesthesia records that did not correspond to the reporting form and resulted in subsequent random chemical analysis of waste drugs. No drug diversion was suspected. The authors note successes in the program were due to assigning a unique identifier number to each drug container, and proper education of staff. Both of these early studies examined controlled substances (opioid) accounting practices, and laid the foundation for current opioid control processes in health care systems.

Vigoda, Gencorelli, and Lubarsky (2007) did a retrospective study reviewing anesthesia records for documentation of four common medications (opiates, benzodiazepines, ketamine, and thiopental) used during anesthesia and compared this data with that of medications dispensed from a automated medication dispensing system. Statistical linear regression analysis was used, which was appropriate, and significance was achieved with the results. They found that $15 \%$ of cases had a discrepancy between what was dispensed versus what was administered, and waste 
was frequently incorrectly recorded. Investigators noted data entry errors in both administration and dispensing data, limiting the utility of automating medication reconciliation for atypical drug patterns. This paper supports the earlier work (Kamerman, Quirk, \& Bluml, 1992; Schmidt \& Schlesinger, 1993) suggesting that poor record keeping and data entry errors account for medication discrepancies, as opposed to diversion or loss.

Most recently, McClure, O’Neal, Grauer, Couldry, and King (2011) conducted a descriptive study, utilizing an investigator-developed questionnaire to survey a national sample of hospital pharmacy directors about their practices for detection of controlled substance diversion in their institution. Descriptive statistics were used, and correlational-relationships were analyzed using chi-square and Fisher's exact tests, which were appropriate for this ordinallevel of data. The findings showed that $97 \%$ of institutions surveyed use decentralized automated drug-dispensing machines, but did not have such a machine within the central pharmacy. Many institutions reported utilizing cameras (65\%), auditing the use of and waste procedures of controlled substances (85\%), and auditing person-to-person transactions (70\%). Half had operating room satellite pharmacies. However, many report having no procedures to verify a drug's identity. And 66\% do not audit non-controlled medications against dispensation. A limitation of low response rate was noted and could have introduced nonresponse bias and affected the quality of the results.

The remaining articles found were case studies describing how individual health care facilities have approached the issue of controlled substance and/or propofol accountability. For example, Mayo Clinic describes efforts to improve education about risks of drug diversion, institution of active surveillance for atypical patterns, evaluation of instances of potential diversion, and development of strategies for a uniform response to suspected diversion (Berge, 
Dillon, Sikkink, Taylor, \& Lanier, 2012). Le Bonheur Children's Medical Center in Tennessee describes improvement of policies, aggressive investigations, and tougher enforcement to address drug diversion in their institution (Berry \& Bogardus, 2000). Beth Israel Hospital in Boston began an in-service program to educate hospital staff about drug diversion to heighten awareness (Carlson, Castile, \& Janousek, 1988). Children's Mercy Hospitals and Clinics in Kansas City assembled a team to look at current practices surrounding controlled substances and then to develop, implement, and evaluate a system to eliminate improper access to controlled substances within their institution (Hulse, Edmundson, \& Carroll, 2004). A hospital in Calgary reported their implementation of dispensing controlled substances in kits to improve accountability (Maltby, Levy, \& Eagle, 1994). New Haven Hospital described a satellite pharmacy in the operating room to increase control and accountability of all drugs (Moleski, Easley, Barash, Primer, Shier, \& Schrier, 1985). Cleveland Clinic instituted an active diversion and substance abuse program, with education programs, procedures to detect diversion, and enhanced measures to detect impairment (Tetzlaff, Collins, Brown, Pollock, \& Popa, 2010). Finally, the Minnesota Hospital Association (2015) took at strong stance to some cases of provider or staff substance abuse and drafted a detailed handbook to dealing with the diversion issue. The majority of these authors report having addressed the drug diversion problem in their institution in response to cases of drug diversion or provider substance abuse in their facility.

Across these case studies, similar approaches were instituted, including staff education, updated and strengthened policy, use of surveillance systems, and active investigation of suspected cases of diversion. Although described as useful in these institutions, case studies have a risk of bias and are less generalizable to a broader group of people. Implementing these 
same strategies in other institutions may not be successful because of lack of automated systems, lack of resources, or lack of manpower (Melnyk \& Fineout-Overholt, 2011).

To summarize, while there is consistency in findings across study designs, the general level of evidence found in the studies of expert opinion and case studies is considered weak, and therefore it is difficult to determine their validity and generalizability. All the articles describe the practice setting as a health care facility, most within an operating room and involving health care workers within an anesthesia department, although one setting specifically addresses nurses in a unit setting. Although all articles addressed the methods used to track drugs specifically in their institutions, all echoed similar themes: controlled substances or drugs with the potential of abuse need to have stricter accountability and regular examination of patterns of handling these drugs can reveal problems. The articles suggest that continued programs and research studies should be completed to deal with drug diversion.

\section{Rationale for Project}

Different studies described many of the same solutions to the problem of controlled substance drug diversion: satellite pharmacies, automated drug dispensing systems and/or anesthesia management systems, drug kits, drug diversion surveillance, and formal educational programs- all to strengthen controls of drug access. However, there were clear gaps in the literature on universal best practices, with no guidance on propofol specifically. Therefore, this study implemented and evaluated a new method of tracking propofol, with the expectations that these changes would improve accountability, provide consistent inventory, decrease loss, improve billing capture, and conceivably decrease the risk for diversion. The project addressed evidence-based calls for stricter accounting of propofol in clinical practice, and was based upon the evidence reviewed, personal clinical observations, professional association guidelines 
(American Association of Nurse Anesthetists, 2012; American Society of Anesthesiology, 2013), and feedback from key providers involved in propofol administration and reconciliation.

While planning this EBP project, it was important to prospectively identify positive and negative factors to implementing the overall project plan. A helpful tool in assessing the uncertainty of success for a given intervention was the SWOT (Strengths-WeaknessesOpportunities-Threats) analysis (Poe \& White, 2010). Utilizing this tool in the planning stage helped highlight the potential barriers and facilitators to the proposed clinical practice change. The analysis revealed there are more strengths and opportunities than weaknesses or threats to implementing this project. Because the health care institution and the anesthesia community value patient safety, initiatives to improve safety processes were well received. Potential regulatory compliance issues also drove the project change. However, barriers such as cost and frequent introduction of new providers in the GI Endoscopy unit were problems that will need to be addressed in the future. 
Table 2. SWOT analysis.

\begin{tabular}{|c|c|}
\hline STRENGTHS & WEAKNESSES \\
\hline $\begin{array}{l}\text { - Culture of safety in institution } \\
\text { - Team consists of professional experts } \\
\text { in drug management- Pharmacy } \\
\text { - Existing processes/ technology in } \\
\text { place } \\
\text { - PI has good working relationship with } \\
\text { anesthesia department } \\
\text { - PI has credentials to work at } \\
\text { institution/ "insider" }\end{array}$ & $\begin{array}{l}\text { - PI has no prior working relationship } \\
\text { with pharmacy } \\
\text { - Cost restraints } \\
\text { - Many anesthesia trainees who are not } \\
\text { familiar with institutional processes } \\
\text { and policies }\end{array}$ \\
\hline OPPORTUNITIES & THREATS \\
\hline $\begin{array}{l}\text { During a recent review, the } \\
\text { Department of Health requested a } \\
\text { practice change for propofol } \\
\text { - Potential to improve process prior to } \\
\text { implementation of anesthesia AIM } \\
\text { and incorporate changes as needed } \\
\text { - Increased leadership interest due to } \\
\text { past personnel substance abuse }\end{array}$ & 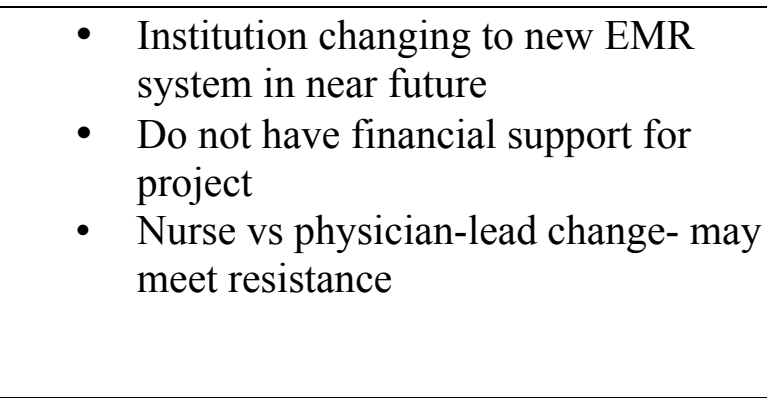 \\
\hline
\end{tabular}




\section{Chapter III}

\section{Methodology}

The goal of this project was to implement a new method of tracking propofol and evaluate efficacy of improved accounting with respect to decreasing propofol loss and associated cost to the institution with lost billing capture. Over a 2-week observation period, data describing current practice was collected, including amounts of drug dispensed, administered, wasted, returned, and billed. From these, measures of unaccounted for medication, plus associated costs, were calculated. Grounded in evidence, a "best-practice" pharmacy process change leading to a new policy was then constructed and formally documented (see Appendix B). A series of educational sessions occurred, with anesthesia, nursing, pharmacy, and other members of the GI Endoscopy Unit, describing the new tracking process. The process was then instituted, and the change in practice process by anesthesia personnel evaluated, to include better documentation of propofol usage and waste and more transparent tracking of the drug throughout the unit. After a five month implementation period, post-intervention data was collected over an additional 2week observation period and analyzed compared to pre-implementation measures to ascertain if improvements in tracking, and therefore decrease in propofol loss and improved billing, did occur.

\section{Research Design}

A prospective descriptive study using a quasi experimental pre-post design was conducted to compare the handling of propofol in a single gastrointestinal endoscopy unit immediately before, and five months following, a policy change that modified the process of how the drug propofol is dispensed, wasted, and/or returned. Over two, 2-week observation 
periods, data was extracted from the Pyxis Medstation or a pharmacy medication inventory system (Kit Check®, Kitcheck, Inc.), paper anesthesia records, and paper pharmacy billing sheets using a data collection guide (see Appendix C). Variables measured include (a) amount of propofol inventoried in either the Pyxis Medstation or Kit Check system; (b) amount of propofol dispensed per provider and patient name associated with the transaction; (c) amount of propofol administered to each patient; (d) amount of propofol returned to the Pyxis Medstation or Kit Check system; (e) amount of propofol recorded on the pharmacy billing sheet; and (f) amount of propofol inventoried in Pyxis Medstation or Kit Check system at the end of day. This data was then added or subtracted to determine frequencies and percentages of cases that accounting or billing was correct.

A process change and policy was developed based on best evidence and pre-data with input from a multidisciplinary team of anesthesia and pharmacy members (see Appendix B). The policy outlined a change in propofol storage from the Pyxis Medstation to procedure-roombased anesthesia lockable carts. Inventory was tracked in the pharmacy with a Kit Check system, pharmacy-processing software that tracks medication. Policy information was disseminated to anesthesia, pharmacy, and nursing staff via educational sessions consisting of lectures, emails, and poster reminders. Outcome measures included amount of propofol loss and costs associated with loss.

\section{Project Sponsors}

A clinical champion on the GI Endoscopy Unit monitored compliance with the new policy and process change, and continues to ensure the longevity of the project by supporting this project on a regular basis. This champion has extensive institutional knowledge, having practiced at MedStar Georgetown University Hospital since 1986 and holding the title of 
Director of GI Anesthesia. An active clinical researcher, he served as a powerful presence for the project.

\section{Procedures/Timeline}

Data was collected over two 2-week observation periods. Data was extracted from either the Pyxis Medstation or Kit Check system, paper anesthesia records, and paper pharmacy billing sheets using a data collection guide (see Appendix C). Propofol inventory on this unit consists of individual 20 -mL vials containing $200 \mathrm{mg}$ of drug. After the first data collection period, a new process and policy, crafted by a multidisciplinary team from anesthesia, pharmacy, and nursing, was implemented to track propofol inventory. Specifically, propofol storage changed from the automated medication management system (Pyxis Medstation) to procedure-roombased anesthesia lockable carts. Inventory was tracked in the pharmacy with Kit Check systempharmacy processing software, instead of the Pyxis Medstation. Five months postimplementation, data was collected again over a 2-week observation period utilizing the same procedures.

Educational sessions on the new process and policy were delivered to anesthesia department members prior to implementation. The sessions included a power point presentation delivered by the researcher, detailing the aims of this project and the practice changes related the new policy. The sessions included opportunity for comments and feedback by providers.

Educational sessions were followed by email blasts and reminder messages. Signage was added to anesthesia carts to outline important steps to remember.

This project took a total of nine months to complete. Specifically following IRB approval, 14 days were required to collect baseline data, 4 weeks for data analysis, 12-14 weeks for process and policy development, and 10 days for educational in-services. Post-intervention 
data collection was completed in July 2016, at which time comparative data analysis proceeded and dissemination activities were initiated. Data entry occurred concurrently with project implementation and pre-intervention data was subject to interim analysis.

Data was collected in a Microsoft Excel spreadsheet. The researcher has maintained security by keeping the document password protected on a single personal computer and stored in a locked office. Data will be available for a period of 3 years, per IRB protocol, and then destroyed.

\section{Instrument}

Data was extracted from the Pyxis Medstation or Kit Check system, paper anesthesia records, and paper pharmacy billing sheets over a 2-week observation period at both time points using a validated data collection guide consisting of fifteen items, categorized by demographics and case type (4 items), propofol amounts that were inventoried, administered, returned (10 items), and propofol amounts billed (1 item) (see Appendix C). The first section of the instrument containing four patient demographics and case type questions was designed to obtain information about age of patient, type of procedure performed, procedure room number, and gender of the patient. The second section of the guide was comprised of ten questions documenting the quantity of propofol accounted for at different stages of handling in the GI Endoscopy Unit, including amounts inventoried in the automated medication management system, administered to the patient, and returned to the automated medication management system or drug return bin. The guide concluded with one question pertaining to pharmacy billing and records the propofol amounts billed (see Appendix D).

The researcher developed the 15- item data collection guide, with four content experts from pharmacy and anesthesia departments evaluating the instrument for content validity. The 
expert reviewers were asked to evaluate the instrument for relevance, clarity, and overall usefulness; and to provide written feedback. The expert's feedback was used to strengthen the data collection guide before using it. Two content experts were pharmacy leadership, and two were anesthesia department leadership, all practicing in the institution where the project takes place.

\section{Protection of Human Subjects}

No provider or patient identifiers were recorded. Permission to conduct this study was approved by the Georgetown University Institutional Review Board, which determined that the study protocol was exempt from full board review and thus waived any requirement for consent (IRB no. 2015-0956).

\section{Population Sample}

Cases of patients undergoing colonoscopy (CLN), esophagogastroduodenoscopy (EGD), endoscopic ultrasound (EUS) procedures, and small bowel balloon enteroscopy performed on the GI Endoscopy Unit requiring propofol sedation were interrogated for two 2-week observation periods prior to and following the intervention. Sample size was calculated on an $80 \%$ power level and the ability to detect a significant $(\mathrm{p}<0.05)$ improvement in propofol tracking assuming a $20 \%$ rate of unaccounted for propofol before the policy change and a $50 \%$ reduction of this rate after the policy change. This yielded a sample size of $n=196$ for each time period (pre- and postpolicy change) for a total of 400 cases that were to be analyzed for this project. Exclusion criteria included all endoscopic retrograde cholangiopancreatography (ERCP) procedures, bronchoscopy procedures, and patients $<18$ years old. Rationale for exclusions included differing anesthesia techniques, non-gastrointestinal tract procedures, or special populations such as extremes in age. 


\section{Outcome Measures/Data Analysis Plan}

Data from this study was analyzed using Statistical Packages for Social Sciences (SPSS) software version 23. Descriptive statistical methods were used to analyze the frequency distribution of demographic data including age, gender, and case type. To evaluate propofol loss and associated cost, several descriptive outcome measures were calculated and compared preand post-intervention, including (a) percentage of cases for which propofol was dispensed; (b) percentage of cases the amount of propofol dispensed matched what was administered, wasted and/or returned; (c) percentage of cases that had $>200 \mathrm{mg}$ (one 20-mL vial) of propofol unaccounted (dispensed, but not administered or returned); (d) total volume of propofol missing from accounting (inventoried minus dispensed, administered, or wasted); (e) percentage of cases with a missing pharmacy billing form; and (f) percentage of cases that did not documented billing for propofol on the pharmacy billing form. In addition, exploratory correlational analysis was completed to determine if there is a relationship pre- and post-intervention between total amount missing from accounting by comparing the variables amount dispensed, administered, wasted, and returned; case type and amount of propofol dispensed; and case type and having $>200 \mathrm{mg}$ (one 20-mLvial) of propofol unaccounted. To calculate cost, the project site pharmacy purchases each 20 -mL propofol vial for $\$ 2.10$, or $\$ 0.105 / \mathrm{mL}$. 


\section{Chapter IV}

\section{Results}

\section{Analysis of Data}

The study was designed to determine the impact of an evidence-based process change on propofol accounting, specifically loss and billing capture. Data was collected before and after the implementation, and changes in outcome measures evaluated for statistical significance using chi-square and correlational analysis. Descriptive statistical methods were used to analyze frequency distribution of demographic data. Exploratory inferential analysis was completed with t-test and chi square testing to determine if there is a relationship between pre- and postimplementation phases.

The sample included all GI Endoscopy cases meeting study inclusion criteria that had received propofol sedation over two 2-week observation periods prior to and following implementation. Using the data collection guide (see Appendix C), data from the Pyxis Medstation or Kit Check system, anesthesia patient records, and pharmacy billing sheets was gathered. As noted, during the course of the study, a new pharmacy inventory system (Kit Check ${ }^{\circledR}$, Kitcheck, Inc.) combined with drug trays in procedure room anesthesia carts replaced the original Pyxis Medstation; this prevented the collection of the variables (a) amount dispensed (b) amount returned in the post-implementation phase, but added the variable of waste which was not available in the pre-implementation phase. In addition, a remodeled pharmacy billing form that included documentation of propofol waste was instituted in the post-implementation phase (see Appendix D). 
As presented in Table 3, in the pre-implementation phase 300 cases were reviewed and in the post-implementation 308 cases were reviewed, providing adequate statistical power. No statistically significant differences were noted in patient gender, age, or case type between observation periods, highlighting the effect of the intervention on the outcomes. The sample reflected males and females equally, with the mean age of 56.4 years old $(\mathrm{SD}=15.1)$. Propofol was used in a variety of GI endoscopy procedures, with both phases of the project reporting colonoscopy as the most frequently performed procedure, followed by upper endoscopy, combined colonoscopy/upper endoscopy, and upper endoscopy with ultrasound. 
Table 3. Sample and case demographics, pre- and post-intervention.

\begin{tabular}{|c|c|c|c|c|}
\hline & $\begin{array}{l}\text { Pre- } \\
\text { implementation } \\
\text { phase }(n=300)\end{array}$ & $\begin{array}{l}\text { Post- } \\
\text { implementation } \\
\text { phase }(n=308)\end{array}$ & \multicolumn{2}{|l|}{$\begin{array}{l}\text { Total } \\
(n=608)\end{array}$} \\
\hline \multicolumn{5}{|l|}{ Gender } \\
\hline No. Female (\%) & $159(53 \%)$ & $144(47 \%)$ & \multicolumn{2}{|l|}{$303(50 \%)$} \\
\hline Age (mean, SD) & $57(15.1)$ & $56(15.3)$ & \multicolumn{2}{|l|}{$56.4(15.2)$} \\
\hline Case types & & & Total & $\begin{array}{l}\text { Propofol } \\
\text { administered } \\
\text { per case }\end{array}$ \\
\hline CLN & $111(37 \%)$ & $129(42 \%)$ & $240(40 \%)$ & $\begin{array}{l}395 \mathrm{mg} \\
(\mathrm{SD}=157)\end{array}$ \\
\hline EGD & $80(27 \%)$ & $88(29 \%)$ & $168(28 \%)$ & $\begin{array}{l}357 \mathrm{mg} \\
(\mathrm{SD}=163)\end{array}$ \\
\hline EGD/CLN & $34(11 \%)$ & $44(14 \%)$ & $78(13 \%)$ & $\begin{array}{l}542 \mathrm{mg} \\
(\mathrm{SD}=224)\end{array}$ \\
\hline $\begin{array}{l}\text { Enterscopy } \\
\text { Single balloon Upper }\end{array}$ & 1 & 1 & $2(0.3 \%)$ & $\begin{array}{l}838 \mathrm{mg} \\
(\mathrm{SD}=53)\end{array}$ \\
\hline EUS & $62(21 \%)$ & $34(11 \%)$ & $96(16 \%)$ & $\begin{array}{l}413 \mathrm{mg} \\
(\mathrm{SD}=179)\end{array}$ \\
\hline Flex sig & $3(1 \%)$ & 0 & $3(0.5 \%)$ & $\begin{array}{l}293 \mathrm{mg} \\
(\mathrm{SD}=51)\end{array}$ \\
\hline Ileo & $8(3 \%)$ & $11(4 \%)$ & $19(3.1 \%)$ & $\begin{array}{l}184 \mathrm{mg} \\
(\mathrm{SD}=95)\end{array}$ \\
\hline Ileo/EGD & 0 & 1 & $1(0.2 \%)$ & $500 \mathrm{mg}$ \\
\hline Insertion G-tube & 1 & 0 & $1(0.2 \%)$ & $350 \mathrm{mg}$ \\
\hline Total & & & $\begin{array}{l}608 \\
(100 \%)\end{array}$ & $\begin{array}{l}401 \mathrm{mg} \\
(\mathrm{SD}=184)\end{array}$ \\
\hline
\end{tabular}

Over the four weeks (two x 2-weeks) of observation period, the total amount of propofol administered in the GI endoscopy unit was 243,286 mg (@1217 20-mL vials). The daily average amount of propofol administered per case (regardless of type) was 401mg (approximately 220 $\mathrm{mL}$ vials), with single-balloon upper enteroscopies requiring the highest average amount of 
propfol at $837 \mathrm{mg}$, followed by EGD/CLN with $542 \mathrm{mg}$ and ileo/EGD cases with $500 \mathrm{mg}$. The case type requiring the least amount of propofol on average was ileoscopies with $184 \mathrm{mg}$.

\section{Propofol Accounting}

Across these cases, a total of 239,200 mg (@119620-mLvials) of propofol was inventoried in the pre-intervention period and 356,400 mg (@1782 20-mLvials) postintervention period (see Table 4).

Table 4. Propofol accounting and loss, pre- and post-intervention.

\begin{tabular}{|l|c|c|c|}
\hline & $\begin{array}{l}\text { Pre-implementation } \\
\text { phase (n=300) }\end{array}$ & $\begin{array}{l}\text { Post-implementation } \\
\text { phase (n=308) }\end{array}$ & Significance \\
\hline Amount dispensed & $190,399 \mathrm{mg}$ & $198,000 \mathrm{mg}$ & n.s. \\
\hline Amount administered & $119,836 \mathrm{mg}$ & $123,450 \mathrm{mg}$ & n.s. \\
\hline Amount returned & $24,888 \mathrm{mg}$ & unknown & \\
\hline Amount wasted & unknown & 15,800 & $\mathrm{n} . \mathrm{s}$ \\
\hline $\begin{array}{l}\text { Amount unreconciled } \\
\text { (loss) }\end{array}$ & $58,704 \mathrm{mg}$ & $51,462 \mathrm{mg}$ & n.s \\
\hline$\%$ unreconciled & $31 \%$ & $26 \%$ & \\
\hline
\end{tabular}

Amounts dispensed (190,399 mg pre- vs. 198,000 mg post-implementation) and administered (119,836 mg pre- vs. $123,450 \mathrm{mg}$ post-implementation) did not differ significantly. Although not directly comparable, the amounts returned in the pre-implementation phase was $15,800 \mathrm{mg}$ and amounts wasted in the post-implementation phase was $24,888 \mathrm{mg}$. To evaluate if the propofol inventory intervention improved reconciliation, the amount of medication dispensed was compared to that documented as administered, wasted or returned for each observation period. The percentage of cases for which propofol accounting was complete was $71 \%$ prior to intervention vs. $75 \%$ post intervention, reflecting a slight improvement, which was not statistically significant.

The approximate percentage of cases in which accounting was not complete decreased between the pre and post implementation periods. Over the initial pre-implementation period, 
$58,704 \mathrm{mg}$ of propofol were unaccounted for, representing a loss of $31 \%$ of the propofol inventory. Ten-percent $(\mathrm{n}=31)$ of the cases did not have propofol signed out of the Pyxis Medstation even though the correlating anesthesia record documented propofol having been administered and/or billing for it on the pharmacy form was absent. Data also revealed $25 \%$ ( $\mathrm{n}=$ 74) of cases had greater than $200 \mathrm{mg}$, the equivalent of $120-\mathrm{mL}$ vial, signed out of Pyxis Medstation but not recorded as administered or returned, suggesting that extra vials of drug were removed from Pyxis Medstation. Of note, amount of propofol was documented only in the preimplementation phase, and was $15,800 \mathrm{mg}$ for the 2-week period. Amount of propofol wasted was documented only in the post-implementation phase, and was $24,888 \mathrm{mg}$ for the 2-week period. With the new process of accounting in place post-implementation, $51462 \mathrm{mg}$ of propofol were unaccounted for (removed from tray but not recorded as administered or recorded as waste). This created a smaller loss of drug of $26 \%$ missing from inventory (see Table 4 ).

\section{Propofol Billing and Cost}

To determine whether the new method of tracking and accounting for propofol inventory improved billing capture, cases which were correctly billed on the pharmacy billing sheet were identified. In the pre-implementation phase, $83 \%(n=200)$ of the cases were correctly billed. The post-implementation phase showed improvement with $88 \%(n=256)$ of cases receiving correct billing; however the change did not meet statistical significance (see Table 5). Next cases that did not submit a billing sheet were analyzed. Pharmacy billing for propofol preimplementation revealed that $20 \%(\mathrm{n}=59)$ of cases had no pharmacy billing sheet available, despite the recording of propofol administration on the anesthesia record. Another $1.7 \%(n=5)$ of cases returned the billing form without recording of an amount of propofol used. Therefore, no propofol billing occurred in almost one-quarter of cases in the pre-implementation phases. In the 
post-implementation phase, the percentage decreased to $6 \%(\mathrm{n}=18)$, demonstrating a statistically significant improvement in billing $(\mathrm{p}=<0.000)$. The number of cases of billing sheets returned to pharmacy with no recording of the amount used increased slightly after the invention (1.7\% to $2.6 \%, p=$ n.s.)

Table 5. Pharmacy billing, pre- and post-intervention.

\begin{tabular}{|l|c|c|c|}
\hline & $\begin{array}{l}\text { Pre-implementation } \\
\text { phase (n=300) }\end{array}$ & $\begin{array}{l}\text { Post-implementation } \\
\text { phase (n=308) }\end{array}$ & Significance \\
\hline $\begin{array}{l}\text { No. Correct billing } \\
\%\end{array}$ & $200(83 \%)$ & $256(88 \%)$ & n.s. \\
\hline $\begin{array}{l}\text { No. No billing sheet } \\
\%\end{array}$ & $59(20 \%)$ & $18(6 \%)$ & $\mathrm{p}<0.00$ \\
\hline $\begin{array}{l}\text { No. incomplete } \\
\text { billing sheet } \\
\%\end{array}$ & $5(1.7 \%)$ & $8(2.5 \%)$ & n.s. \\
\hline
\end{tabular}

\section{Summary of Findings/Outcomes}

These analyses suggest that although propofol, patient, and case characteristics remained constant over time, the new method of tracking and accounting for propofol inventory in GI endoscopy unit decreased the amount of "unaccounted for" or missing drug as evidenced by less loss of inventory and increased billing capture with the submission of a higher percentage of useable pharmacy billing forms. 


\section{Chapter V}

\section{Discussion}

\section{Discussion of Findings}

Drug control is important within health care facilities. Accurate inventory processes ensure correct accounting, a measure needed to guarantee drug availability for patient care and prevent shortage of drugs when purchasing issues arise. Precise control can also aid in investigating lost drug or unnecessary wasting of drug, and therefore decrease costs to the institution. Of particular concern is the accounting of the anesthetic drug propofol, since evidence suggests a strong link to misuse, diversion and abuse among health care professionals.

To address the issues associated with lax accounting, this project's aim was to determine if a new methodology would help with more accurate tracking of propofol on one hospital unit, to prevent loss and capture cost. Previous research supports this approach. Welliver, Bertrand, Garza, and Baker (2012), in their report Two New Case Reports of Propofol Abuse and a Pattern Analysis of the Literature, recommend accountability policies with restrictions to access and signature accountability, but no specific method is described. Vigoda et al. (2007) in their report Discrepancies in Medication Entries between Anesthetic and Pharmacy Records Using Electronic Databases recommended better reconciliation by aligning use between an automated medication dispensing system and an anesthesia patient record database (Vigoda, Gencorelli \& Lubarsky, 2007). And New (2014) in Preventing, Detecting, and Investigating Drug Diversion in Health Care Facilities advocates for policies and procedures addressing the tracking of controlled substances from receipt of disposition, including secure storage prior to use and access restricted to authorized individuals (New, 2014). 
The data for this project suggests that the new system of propofol storage in anesthesia carts and inventory accounting by a pharmacy system was successful in accounting for higher percentage of propofol, but not to a significant degree. The change was only statistically significant in the case of billing. The fact the process change did not have a bigger impact was not surprising. There was culture resistance to tracking propofol because as a uncontrolled substance, regulations do not require it. Despite the small accounting successes, there were breakdowns to the process within the institution post-implementation and the change was not sustained long-term. The project required an operational modification in pharmacy processes, which proved too difficult to continue. While this study builds upon evidence in the literature, highlights the utilization of good hypotheses, and employed the scientific method to achieve a needed quality improvement process, missing was the necessary continued buy-in of all of the stakeholders to sustain the change.

Accounting and Inventory. Within health care facilities, propofol storage, accounting, and security is less stringent than that for other drugs with abuse potential, such as opioids and benzodiazepines (New, 2014). Lax accounting enables easy access to the medication for patient care, but can carry legal and/or regulatory risks associated with management liability from Departments of Health, the Joint Commission, and/or law enforcement. Operational consequences include inconsistent inventory and unaccounted for drug costs.

The process change for this project involved no longer using a centrally located Pyxis Medstation but instead utilizing individual anesthesia carts found in each procedure room. This change was contrary to suggestions from drug diversion studies that envisioned automated medication systems as a superb method of tracking drugs (Epstein, Gratch \& Grunwald, 2007; Epstein, Gratch, McNulty, \& Grunwald, 2011; Vigoda, Gencorelli, \& Lubarsky, 2007). The 
Pyxis Medstation in the GI unit operated as a secured storage bin only, with little reconciliation occurring between what was removed and administered; nor was billing generated from the system. No wastage was recorded in any of the documentation. The change of stocking propofol in drugs trays improved provider efficiency but necessitated a different accounting system. Pharmacy instituted an in-pharmacy inventory system (Kit Check) to count drug removed from trays. A new billing form was also introduced to record waste of drug.

Accounting for individual vials of drug slightly improved following implementation of the new process. This system is dependent on providers' filling out and recording accurately propofol administration and wastage on a form in addition to the written anesthesia record. Previous researchers have reported on notable documentation errors and omissions limiting accounting in their studies and advocate automated record keeping to improve data entry (Kamerman, Quirk, \& Bluml, 1992; Schmidt, \& Schlesinger, 1993). Pre-intervention, providers for this project may have understood billing to occur from Pyxis records, which was false; without Pyxis recordings in the post-intervention phase, providers may have felt it more necessary to complete the billing form that lead to improved accounting. Waste was not recorded in any manner in the pre-intervention phase. In the post-intervention phase, this data was captured and could be used for reconciliation. With the new system, it would be nearly impossible to track drug handling by provider, since dispensing from a room's anesthesia cart cannot be tagged to an individual provider, since room assignments on a given day are not consistent.

The literature is limited in reporting on effective ways to handle propofol, particularly in regard to security, access, and accountability. The American Association of Nurse Anesthetists and American Society of Anesthesiology, the professional organizations of nurse anesthetists and 
anesthesiologists, have position statements about the importance of securing propofol, yet neither organization outlines best-practices other than a locked system (American Association of Nurse Anesthetists, 2012; American Society of Anesthesiology, 2013). Automated drug dispensing systems with user-identification, drug kits, and operating room pharmacies have been introduced in some facilities, but their effectiveness in accounting of propofol has yet to be proven (Epstein, Gratch, \& Grunwald, 2007; Tetzlaff, et al., 2010). Periodic audits of patient charts to reconcile dispensing records and drug waste are additional methods described in the literature, but have been reported as time-consuming and testing is costly (Berge, Dillon, Sillink, Taylor, \& Lanier, 2012). Most recently a prevention roadmap outlined by the Minnesota Hospital Association (2015), best practices for prevention of diversion, which correlates with accountability, are published to include the use of bar-coding technology, camera surveillance in areas where controlled substances are stored, electronic-lock cabinets, and regular staff education, however the effectiveness of these interventions has yet to be determined. Researchers Epstein, Gratch, McNulty, \& Grunwald in their article Validation of a System to Detect Scheduled Drug Diversion by Anesthesia Care Providers do caution readers that the ability of precise accounting of propofol would be difficult due to large amount of utilization and wastage in anesthetizing areas, the wide variation in dosing requirements, and patterns of use (Epstein, Gratch, McNulty, \& Grunwald, 2011).

Billing and Cost. This study showed an improvement in loss of drug, which had financial implications for the institution. The cost to purchase one 20-mL vial of propofol (brand Diprivan ${ }^{\circledR}$, Fresenius Kabi, Inc.) is $\$ 2.20$, or approximately 10 cents $/ \mathrm{mg}$. While this is a small cost for a unit of drug, the loss of revenue increases with the number of vials unaccounted for. In the two-week pre-implementation phase of this study, the 54,763 $\mathrm{mg}$ of unaccounted propofol 
totaled $\$ 5750$, and in the post-implementation period $56,800 \mathrm{mg}$ of unaccounted propofol totaled $\$ 5680$. For the entirety of the project, $104,425 \mathrm{mg}$ of propofol were unaccounted for, which is likely an underestimated loss of $\$ 131,575$ annually since the study was limited to the GI Endoscopy unit. For the cases with no billing sheets and therefore no patient billing, estimating at least one $20-\mathrm{mL}$ vial of use per patient, $\$ 1,180$ was not billed, bringing the annual total to more than $\$ 200,000$. While it is speculated that some of drug may have been lost to waste, no data were available to support this hypothesis. Unfortunately, the literature is devoid of reporting the potential health care costs specifically from propofol loss.

This study showed that with the new system, a decrease of $5101 \mathrm{mg}$, the equivalent of 26 fewer vials, were not accounted for, meaning more accurate billing capture of approximately $\$ 268$, saving the institution the loss of this revenue. While a modest amount of funds, the unaccounted loss of potential revenue is poor business.

\section{Limitations}

Operational problems within an organization can result from inadequate or failed internal processes, people, or systems that affect the everyday operations of the business (Chassin \& Loeb, 2013). Health service systems, due to their complex, random, and variable activities, experience operational problems. For this project, in the mid-implementation phase, the process of collecting inventory information from the drug trays with the Kit Check system by the pharmacy department failed. This failure was due to many factors, including a loss of a pharmacy employee and an unfortunate computer hacking incident within the health care system. The pharmacy department was eventually able to collect all post-implementation data for this project, but is not dedicated to sustaining the inventory accounting process long-term. This will leave gaps in the propofol accounting process going forward. 
A limitation was the study excluded data from certain case types, specifically ERCP and bronchoscopy cases, and for patients $<18$ years old. At the time of project development, it was thought the nature of the anesthetic delivered during these cases might complicate data collection; however upon reflection it would have been better-captured overall propofol usage if all propofol information on the entire unit were included. The excluded cases amounted to approximately 8-10 cases in each phase of the project.

\section{Implications for Practice}

The information from this study can be used to guide other GI Endoscopy units, clinics, and procedural sites to create effective procedures and policies to track propofol. In the health care environment, where evidence based practice should be a motivator for change, this information may help guide what priority this particular issue is given within the institution. The risk of diversion by health care providers is real, and enforced policies to limit possibilities of this occurring are needed. In addition, compliance with local health agencies and state regulations maintain an institutions reputation.

This study will enhance the current limited body of information related to propofol handling and accountability in anesthetizing locations. The study results showcase that new processes need to be trialed to evaluate best practices and then standardization of practice created.

This study also highlights the real problem with propofol accounting and tracking within health care institutions and that operational breakdowns occur. Organizational culture may need to alter across all disciplines to embrace evidence-based processes need. Leadership needs to invest and supported process changes. Institutional policy needs to be formed and enforced around this. 


\section{Future Recommendations}

While the literature related to propofol misuse and addiction is increasing, and many authors are calling for better security and accounting of propofol, there are gaps in this literature about best-practice for the handling and tracking propofol within a health care institution. Most processes are time consuming and/or costly. A study similar to this one in which room number is recorded as a proxy for provider identification could add additional information concerning atypical usage or loss. Studies like this need to be done and expanded to multi-units, multiinstitutional, and/or across health systems, to create best practices. 


\section{References}

American Association of Nurse Anesthetists (2012, November). Securing propofol: Formerly position statement number 2.14. Retrieved June 30, 2015, from http://www.aana.com/Pages/default.aspx

Alabama Department of Public Health (July, 2012). News release: State committee of public health adds propofol to Alabama controlled substances list. Retrieved October 6, 2016 from https://www.adph.org/news/assets/120727.pdf

American Society of Anesthesiologists (2013, October). Statement on security of medications in the operating room. Retrieved June 30, 2013, from http://www.asahq.org/

American Society of Health-System Pharmacists (2016, March). Current drug shortage bulletin: Propofol Injection. Retrieved March 10, 2016, from http://www.ashp.org/menu/DrugShortages/CurrentShortages/bulletin.aspx?id=573

Bell, D.M., McDonough, J.P., Ellision, J.S., \& Fitzhugh, E.C. (1999). Controlled drug misuse by certified registered nurse anesthetists. AANA Journal, 67(2), 133 - 140-140.

Berge, K. H., Seppala, M. D., \& Schipper, A. M. (2009). Chemical dependency and the physician. Mayo Clinic Proceedings, 84(7), 625-631. doi:10.4065/84.7.625

Berry, M. \& Bogardus, D. (2000). Hospital security and safety management: How one hospital tackles the drug theft/diversion problem head-on. Hospital Security and Safety Management, 20(11), 8-10. (ISSN: 0745-1148)

Carlson, G. M., Castile, J. A., \& Janousek, J. P. (1988). Guidelines for the prevention and detection of controlled substance diversion. Hospital Pharmacy, 23(12), 1057-1059. 
Center for Medicare \& Medicare Services (CMS) (2016, March). Get the facts: Diversion of pharmaceutical drugs. Retrieved March 10, 2016, from http://www.drugwarfacts.org/cms/Diversion\#sthash.hObhzVEN.dpbs

Chassin, M. \& Loeb, J. (2013). High-reliability health care: Getting there from here. The Milbank Quarterly, 91(3), 459-490.

Epstein, R. H., Gratch, D. M., \& Grunwald, Z. (2007). Development of a scheduled drug diversion surveillance system based on an analysis of atypical drug transactions. Anesthesia and Analgesia, 105(4), 1053-1060. doi:10.1213/01.ane.0000281797.00935.08

Epstein, R. H., Gratch, D. M., McNulty, S., \& Grunwald, Z. (2011). Validation of a system to detect scheduled drug diversion by anesthesia care providers. Anesthesia \& Analgesia,113(1), 160-164. doi: 10.1213/ANE.0b013e31821c0fce

Gaba, D. M. (2000). Anaesthesiology as a model for patient safety in health care. British Medical Journal, 320(3), 785-788.

Georgia Drugs and Narcotics Agency (2016). Georgia law: Laws, rules, regulations. Retrieved October 6, 2016 from https://gdna.georgia.gov/

Haller, G. (2013). Improving patient safety in medicine: is the model of anaesthesia care enough? Swiss Medical Weekly, 143(w13770), 1-8. doi: 10.4414/smw.2013.13770

Hulse, K., Edmundson, L., \& Carroll, R. (2004). Diversion of controlled substances: A catalyst for change. Journal for Healthcare Quality, 26(5), 6-11.

Hvisdas, C., Lordan, A., Pizzi, L. T., \& Thoma, B. N. (2013). US propofol drug shortages: A review of the problem and stakeholder analysis. American Health Drug Benefits, 6(4), $171-175$. 
Jensen, V., \& Rappaport, B. A. (2010). The reality of drug shortages: The case of the injectable agent propofol. The New England Journal of Medicine, 363, 806-807.

Kamerman, R., Quirk, B., \& Bluml, B. (1992). An analysis tool to investigate theft or tampering of controlled substances. Hospital Pharmacy, 27, 223-227.

Lewin, K. (1947). Group decision and social change. In T.N. Newcomb, \& E.L. Hartley (Eds.), Readings of Social Psychology (pp. 459-473). New York: Holt, Rinehart, \& Winston.

Luck, S., \& Hedrick, J. (2004). The alarming trend of substance abuse in anesthesia providers. Journal of PeriAnesthesia Nursing, 19(5), 308-311. doi:10.1016/j.jopan.2004.06.002

Maltby, J. R., Levy, D. A., \& Eagle, C. J. (1994). Simple narcotic kits for controlled-substance dispensing and accountability. Canadian Journal of Anaesthesia. doi:10.1007/BF03009908

McClure, S. R., O'Neal, B. C., Grauer, D., Couldry, R. J., \& King, A. R. (2011). Compliance with recommendation for prevention and detection of controlled-substance diversion in hospitals. American Journal of Health-System Pharmacy,68(4), 689-694. doi:10.2146/ajhp100212

Melnyk, B. M., \& Fineout-Overholt, E. (2015). Evidence-based healthcare. A guide to best practice (3 ed.). Philadelphia: Lippincott Williams \& Wilkins.

Minnesota Hospital Association. (2015). Road map to controlled substance diversion prevention 2.0 (2.0). Retrieved June 24, 2015, from Patient Safety/ Controlled Substance Diversion Prevention website:

http://www.health.state.mn.us/patientsafety/drugdiversion/divroadmap041812.pdf 
Moleski, R. J., Easley, S., Barash, P. G., Primer, G., Shier, N. Q., \& Schrier, R. I. (1985). Control and accountability of controlled substance administration in the operating room. Anesthesia and Analgesia, 64, 989-995. doi:10.1213/00000539-198510000-00008

Monroe, T., Hamza, H., Stocks, G., Scimeca, P. D., \& Cowan, R. (2011). The misuse and abuse of propofol. Substance Use \& Misuse, 46, 1199-1205.

doi:10.3109/10826084.2011.564705

Murray, M. (2001, July). Chapter 11. Automated medication dispensing devices. In A critical analysis of patient safety practices. Evidence report/Technology assessment. Agency for Healthcare Research and Quality, 43. Retrieved from archive.ahrq.gov/clinic/ptsafety/chap11.htm

New, K. (2014). Preventing, detecting, and investigating drug diversion in health care facilities. Journal of Nursing Regulation, 5(1).

Osby, L. (2014, August 20). Drug shortages may be hurting treatment. Greenville Online. Retrieved May 1, 2015, from http://www.greenvilleonline.com/story/news/health/2014/08/20/drug-shortages-putpatient-care-risk/14369115/

Poe, S. S., \& White, K. M. (2010). Johns hopkins nursing evidence-based practice: implementation and translation. Indianapolis, IN: Sigma Theta Tau International.

Roussin, A., Montastruc, J. L., \& Lapeyre-Mestre, M. (2007). Pharmacological and clinical evidence on the potential for abuse and dependence of propofol: a review of the literature. Fundamentals \& Clinical Pharmacology, 21(5), 459-465. doi:10.1111/j.14728206.2007.00497.x 
Schmidt, K., \& Schleshinger, M. (1993). A reliable accounting system for controlled substances in the operating room. Anesthesiology (Philadelphia), 78(1), 184 - 190-190.

Smiledge, P. K., \& Davern, P. F. (1984). An anesthesia controlled substance dispensing system. Hospital Pharmacy, 19(9), 609-613.

Stocks, G. (2011). Abuse of propofol by anesthesia providers: The case for re-classification as a controlled substance. Journal of Addictions in Nursing, 22(1-2), 57-62.

Substance Abuse and Mental Services Administration (SAMSA) (2016, March). Substance use disorders. Retrieved March 10, 2016, from http://www.samhsa.gov/disorders/substanceuse

Tetzlaff, J., Collins, G. B., Brown, D. L., Pollock, G., \& Popa, D. (2010). A strategy to prevent substance abuse in an academic anesthesiology department. Journal of Clinical Anesthesia, 22, 143-150. doi:10.1016/j.jclinane.2008.12.030

Titler, M., Steelman, V., Budreau, G., Buckwalter, K., \& Goode, C. (2001). The Iowa model of evidence-based practice to promote quality care. Critical Care Nursing Clinics of North America, 13(4), 497-509.

The Joint Commission. (2014). The joint commission international accreditation standards for hospitals (5th ed.). Retrieved June 10, 2015, from http://www.jointcommissioninternational.org/assets/3/7/Hospital-5E-Standards-OnlyMar2014.pdf

Vigoda, M. M., Gencorelli, F. J., \& Lubarsky, D. A. (2007). Discrepancies in medication entries between anesthetic and pharmacy records using electronic databases. Anesthesia and Analgesia, 105(4), 10611065. doi:10.1213/01.ane.0000282021.74832.5e 
Welliver, M., Bertrand, A., Garza, J., \& Baker, K. (2012). Two new case reports of propofol abuse and a pattern analysis of the literature. International Journal of Advanced Nursing Studies, 1(1), 22-42.

Wischmeyer, P. E., Johnson, B. R., Wilson, J. E., Dingmann, C., Bachman, H. M., Roller, E., Tran, Z. V. \& Henthorn, T. (2007). A survey of propofol abuse in academic anesthesia programs. Anesthesia \& Analgesia, 105(4), 1066-71.

U. S. Department of Justice: Drug Enforcement Administration. (2010, October 27). Rules 2010 - placement of propofol into schedule IV; proposed rule. Retrieved July 15, 2015, from http://www.deadiversion.usdoj.gov/fed_regs/rules/2010/fr1027.htm

U. S. Food and Drug Administration. (2007, July). Drug approval package: Diprivan.

Retrieved October 5, 2016 from

http://www.accessdata.fda.gov/drugsatfda_docs/nda/2001/019627_S035_DiprivanTOC.c fm 


\section{Appendix A}

\section{Iowa Model of Evidenced-Base Practice to Promote Quality Care}

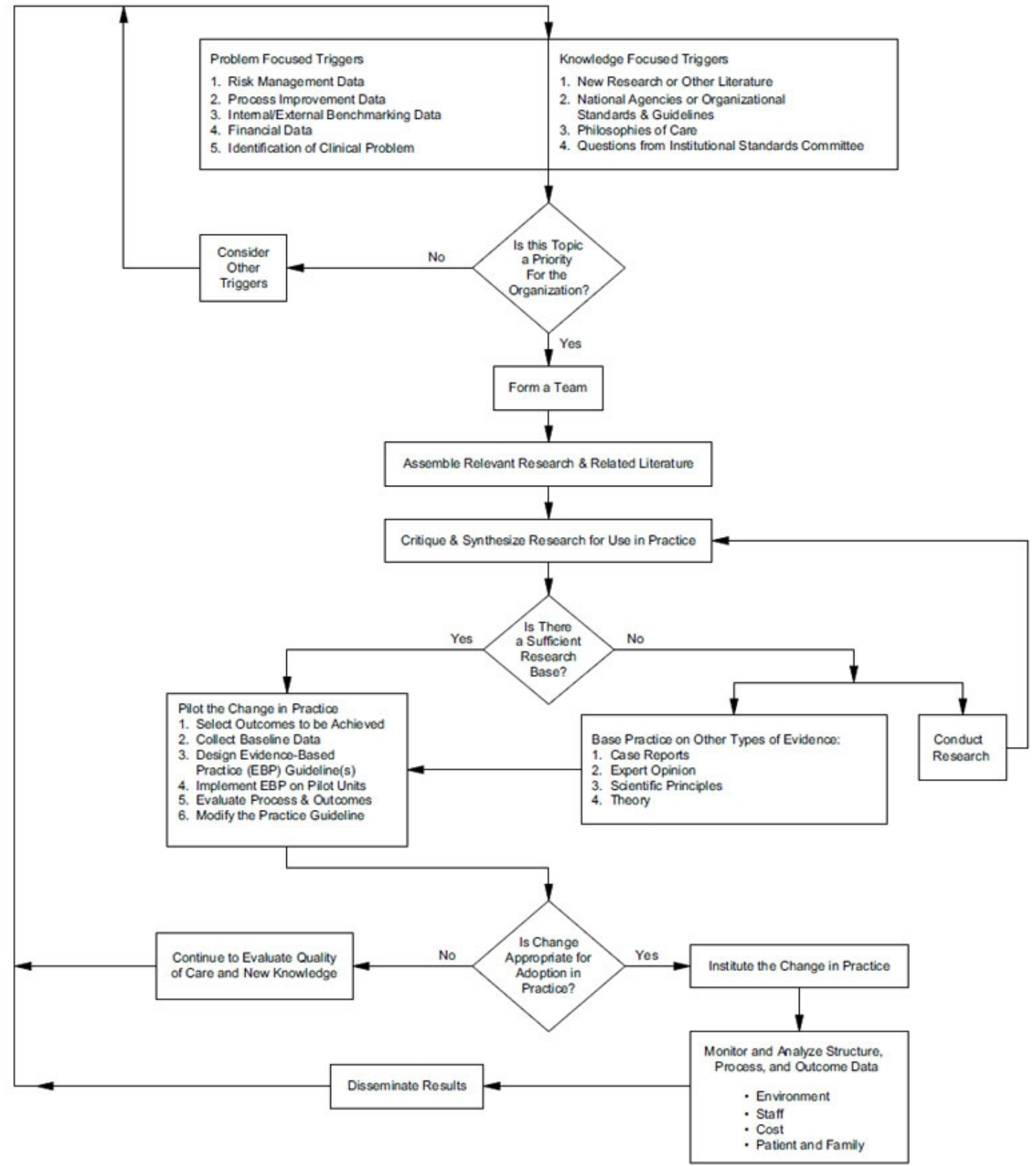

DO NOT REPRODUCE WITHOUT PERMISSION

$$
\begin{array}{r}
\text { REQUESTS TO: } \\
\text { Department of Nursing } \\
\text { The University of lowa Hospitals and Clinics } \\
\text { lowa City, IA 52242-1009 } \\
\text { Revised April } 1998 \text { O UIHC }
\end{array}
$$

Titler, M.G., Kleiber, C., Steelman, V.J., Rakel, B.A., Budreau, G., Everett, L.Q., Buckwalter, K.C., Tripp-Reimer, T., \& Goode C. (2001). The lowa Model of Evidence-Based Practice to Promote Quality Care. Critical Care Nursing Clinics of North America, 13(4), 497-509.

Copyright $\odot 2001$. University of lowa Hospitals and Clinics and Marita Titler. Reproduced with permission from Marita G. Titler, PhD, RN, FAAN. For permission to use or reproduce the model, please contact the University of lowa Hospitals and Clinics, Department of Nursing, at 319-384-9098. 


\section{Appendix B}

\section{GI Endoscopy Unit Propofol Policy}

\section{Propofol Policy GI Endoscopy Unit}

Approved 1/20/16

Accessing propofol in the GI Endoscopy Unit.

As of February 5, 2016, Propofol will be stocked for anesthesia providers in each of the individual anesthesia carts located in the six procedure rooms. When propofol is removed for patient use, a patient identification sticker, amount administered, and amount wasted will be recorded on a revised Pharmacy Billing Form (see attached).

It is imperative that

1. The anesthesia carts be locked, at all times, when an anesthesia provider is not present and, 2. The billing record must be filled out properly and returned to billing file bin with anesthesia record.

Ample propofol will be available in the carts, however if additional vials are required, the automated-drug dispensing system (Pyxis) will be stocked with extra vials. Retrieving drug from the Pyxis requires a proper patient name/ MRN. 
Appendix C

Data Collection Instrument

\begin{tabular}{|c|c|c|}
\hline & Date & Date \\
\hline \multicolumn{3}{|l|}{ Case \# } \\
\hline \multicolumn{3}{|l|}{ Gender } \\
\hline \multicolumn{3}{|l|}{ Age } \\
\hline \multicolumn{3}{|l|}{ Type of Case (CLN, EGD, EUS, other) } \\
\hline \multicolumn{3}{|l|}{ Propofol packet in $\mathrm{mg}$ restocked Pyxis } \\
\hline \multicolumn{3}{|l|}{ Propofol packets in mg in Pyxis } \\
\hline \multicolumn{3}{|l|}{ Propofol in mg in Pyxis or drug tray at start of day (7:00am) } \\
\hline \multicolumn{3}{|l|}{ Propofol in $\mathrm{mg}$ restocked in Pyxis } \\
\hline \multicolumn{3}{|l|}{ Propofol in mg returned to Pyxis } \\
\hline \multicolumn{3}{|l|}{ Propofol in $\mathrm{mg}$ retuned to black return bin } \\
\hline \multicolumn{3}{|c|}{ Propofol in $\mathrm{mg}$ in addition to packets signed out Pyxis per case } \\
\hline \multicolumn{3}{|l|}{ Propofol packets in mg signed out Pyxis per case } \\
\hline \multicolumn{3}{|l|}{ Propofol in mg administered per case } \\
\hline \multicolumn{3}{|l|}{ Propofol in mg documented on billing sheet } \\
\hline \multicolumn{3}{|l|}{ Propofol in mg returned to Pyxis } \\
\hline Propofol in mg documented as wasted on billing sheet & & \\
\hline
\end{tabular}




\section{Appendix D}

\section{Revised Pharmacy Billing Form}

Pharmacy Billing Form

Patient ID sticker

GI Endoscopy Unit

Revised 4/4/16

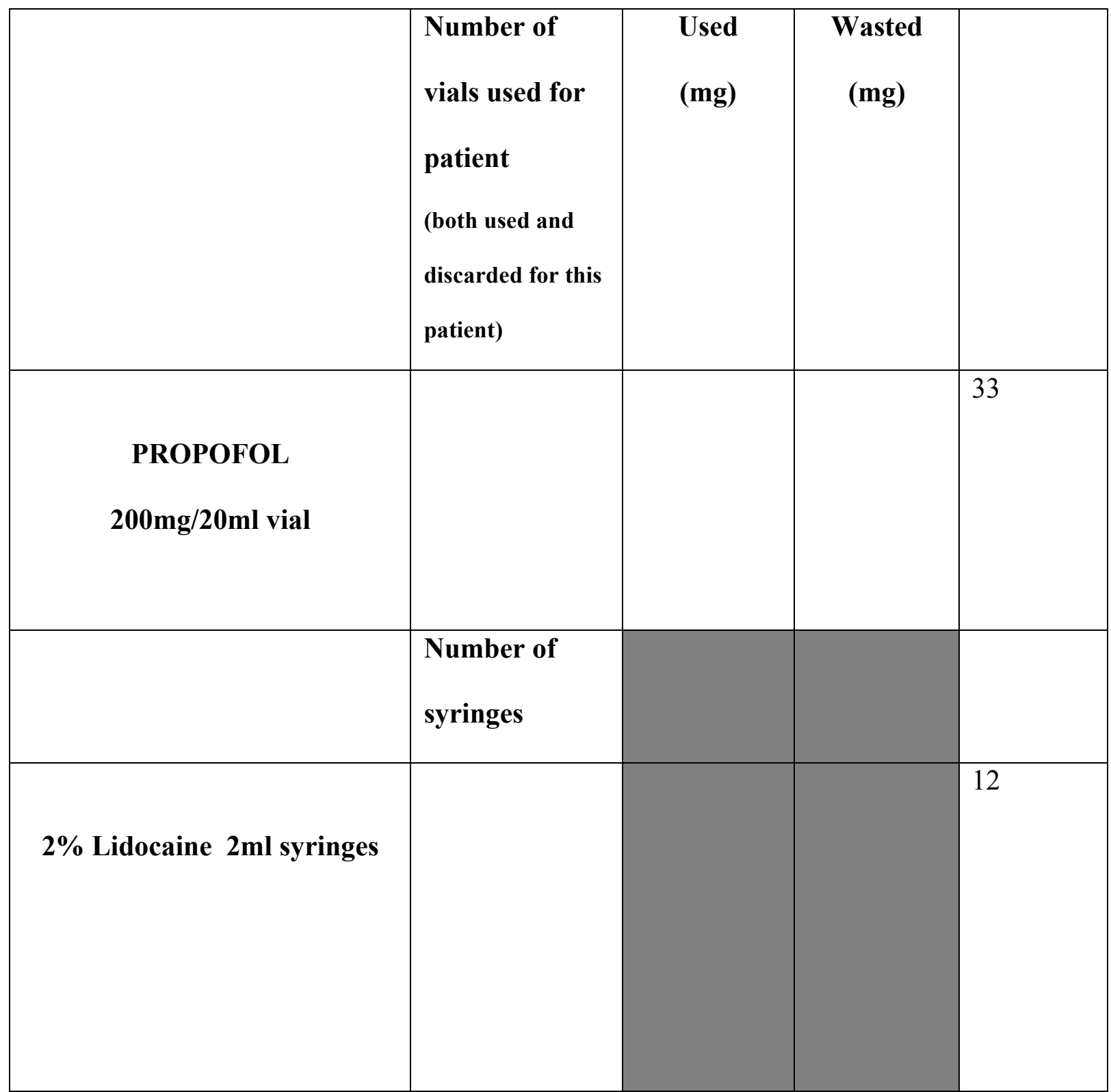


*Please indicate the amount of medication used and return this form to the Pharmacy (to be reconciled with Anesthesia Record)

Administered by

Date

\begin{tabular}{|c|c|c|}
\hline \multirow{14}{*}{$\begin{array}{l}\text { Qty } \\
\text { Used }\end{array}$} & Medication & \\
\hline & Albuterol $90 \mathrm{mcg} / \mathrm{inh}$ inhaler & 1 \\
\hline & Artificial Tears Ointment 3.5g (Lacrilube) & 1 \\
\hline & $\begin{array}{l}\text { Atropine } 0.4 \mathrm{mg} / \mathrm{ml} 2.5 \mathrm{ml} \text { in } 3 \mathrm{ml} \text { pre- } \\
\text { filled syringe }\end{array}$ & 4 \\
\hline & Calcium Chloride $1 \mathrm{gm} / 10 \mathrm{ml}$ Abboject & 1 \\
\hline & Dexamethasone $4 \mathrm{mg} / \mathrm{ml} 1 \mathrm{ml}$ vial & 2 \\
\hline & Diphenhydramine $50 \mathrm{mg} / \mathrm{ml} 1 \mathrm{ml}$ vial & 1 \\
\hline & Ephedrine $10 \mathrm{mg} / \mathrm{ml} 5 \mathrm{ml}$ pre-filled syringe & 4 \\
\hline & Epinephrine $1 \mathrm{mg} / \mathrm{ml} 10 \mathrm{ml}$ Abboject & 1 \\
\hline & Epinephrine $1 \mathrm{mg} / \mathrm{ml} \mathrm{amp}$ & 1 \\
\hline & Esmolol $\mathrm{HCl} 10 \mathrm{mg} / \mathrm{ml} 10 \mathrm{ml}$ vial & 2 \\
\hline & Etomidate $2 \mathrm{mg} / \mathrm{ml} 10 \mathrm{ml}$ vial & 1 \\
\hline & Glucagon $1 \mathrm{mg}$ vial & 0 \\
\hline & $\begin{array}{l}\text { Glycopyrrolate } 0.2 \mathrm{mg} / \mathrm{ml} 5 \mathrm{ml} \text { pre-filled } \\
\text { syringe }\end{array}$ & 2 \\
\hline \multirow{14}{*}{$\begin{array}{l}\text { Qty } \\
\text { Used }\end{array}$} & Medication & \\
\hline & Hydralazine $20 \mathrm{mg} / \mathrm{ml}$ vial & 1 \\
\hline & Hydrocortisone $100 \mathrm{mg}$ vial & 1 \\
\hline & Labetalol $5 \mathrm{mg} / \mathrm{ml} 5 \mathrm{ml}$ syringe & 1 \\
\hline & Lidocaine $2 \%$ jelly $5 \mathrm{ml}$ tube & 2 \\
\hline & Methylprednisolone $125 \mathrm{mg}$ vial & 1 \\
\hline & Metoprolol $1 \mathrm{mg} / \mathrm{ml} 5 \mathrm{ml}$ vial & 1 \\
\hline & Naloxone $0.4 \mathrm{mg} / \mathrm{ml} 1 \mathrm{ml}$ vial & 1 \\
\hline & Ondansetron $2 \mathrm{mg} / \mathrm{ml} 2 \mathrm{ml}$ vial & 6 \\
\hline & $\begin{array}{l}\text { Phenylephrine } 100 \mathrm{mcg} / \mathrm{ml} 10 \mathrm{ml} \text { pre-filled } \\
\text { syringe }\end{array}$ & 4 \\
\hline & Rocuronium $10 \mathrm{mg} / \mathrm{ml} 5 \mathrm{ml}$ vial & 2 \\
\hline & Sodium Chloride USP p.f. $10 \mathrm{ml}$ & 2 \\
\hline & Sterile Water USP p.f. $10 \mathrm{ml}$ & 2 \\
\hline & $\begin{array}{l}\text { Succinylcholine } 20 \mathrm{mg} / \mathrm{ml} 10 \mathrm{ml} \text { pre-filled } \\
\text { syringe }\end{array}$ & 4 \\
\hline
\end{tabular}

